\title{
Application of VIGS system to explore the function of common wheat TaADF7
}

\author{
Huize CHEN $^{1,2, *}$; Xingtian JIA ${ }^{1,2} ;$ HaiYan RAN ${ }^{1,2}$; Jingrong NIU ${ }^{1,2}$; MeIting DU ${ }^{1}$ \\ ${ }^{1}$ Higher Education Key Laboratory of Plant Molecular and Environmental Stress Response, Shanxi Normal University, Linfen, 041000, China \\ 2 School of Life Sciences, Shanxi Normal University, Linfen, 041000, China
}

Key words: Gene silencing, Microfilament binding protein, Crop, Wheat

\begin{abstract}
As an allohexaploid plant, common wheat has a complex gene structure, making it difficult to study its gene function. Virus-induced gene silencing (VIGS) is an important tool for the rapid analysis of plant gene function. In this study, the gene silencing system, namely, barley stripe mosaic virus (BSMV)-VIGS induced by BSMV was used to silence the wheat phytoene desaturase ( $T a P D S$ ) and actin depolymerization factor ( $T a A D F 7)$ genes and determine the effect of gene silencing on wheat. TaPDS was used as an indicator gene to determine the feasibility of VIGS system, while TaADF7 was used as a test gene to determine its effect on wheat growth. Results showed that the leaves of tobacco and wheat were bleached by the mixture of $p C a B S-\alpha, p C a B S-\beta$, and $p C a B S-\gamma:: T a P D S$, indicating that the TaPDS gene was silenced, and the bleached leaves had physiological activity as determined by trypan blue staining. Therefore, the VIGS system was efficient and available. After the tobacco was treated with $p C a B S-\alpha, p C a B S-\beta$, and $p C a B S-\gamma:: T a A D F 7$, the viral suspension was obtained. The expression of TaADF7 gene was downregulated after wheat leaves were infected by friction, indicating that the expression of TaADF7 was silenced. Laser confocal scanning microscopy showed that the silencing of TaADF7 enhanced the fluorescence of microfilament skeleton in mesophyll protoplasts and significantly reduced the plant height. Results showed that TaADF7 affected cell division and plant growth by inhibiting microfilament depolymerization. In conclusion, the BSMV-VIGS system was used to silence wheat TaPDS and TaADF7 genes. Bleaching phenomenon was observed in wheat leaves after TaPDS silencing. After TaADF7 silencing, microfilaments in wheat mesophyll cells gathered into coarse bundles, which affected the dynamics of microfilaments and inhibited plant growth.
\end{abstract}

\section{Introduction}

Common wheat is one of the most important food crops, and it is widely planted worldwide (García-Molina et al., 2019). Common wheat is an allohexaploid plant (AABBDD, $2 \mathrm{n}=$ $6 \mathrm{x}=42$ ), and its genome has the following characteristics: it is composed of three interrelated diploid genomes; the genome is large $(\sim 17 \mathrm{~Gb})$ and is approximately six times that of human genome and 125 times that of Arabidopsis thaliana genome; the proportion of repetitive sequences can reach $80 \%$ and contains many highly homologous sequences; and its transformation is more difficult than that of rice, maize or other crops. Consequently, the analysis of the gene function in common wheat is difficult (El Baidouri et al., 2017; Wang et al., 2015).

*Address correspondence to: Huize Chen, chenhz@sxnu.edu.cn Received: 10 May 2021; Accepted: 07 July 2021
The development of VIGS technology aims to study these complex genomes and plants that involves complicated genetic transformation process (Barciszewska-Pacak et al., 2016). The VIGS technique is to construct single-stranded RNA into viral vectors and infect plants with the virus. Single-stranded RNA is synthesized into double-stranded RNA in plants. Doule-stranded RNA is degraded into small interfering RNA (siRNA) by an enzyme similar to RNAaseIII called Dicer. SiRNA can bind to RNAase to form RNA-induced silencing complex RISC. This RISC complex can specifically attack homologous mRNA and successfully silence the target gene (Lee et al., 2015). PDS is often used as an indicator of whether VIGS is working properly (Zeng et al., 2019). Many viral genomes have been confirmed as VIGS vectors (Unver and Budak, 2009; Becker and Lange, 2010; Senthil-Kumar and Mysore, 2011; Ramegowda et al., 2014). The VIGS system mediated by barley stripe mosaic virus (BSMV) is an effective tool for the gene function analysis of grain and has been widely used in monocotyledon 
studies (Zhang et al., 2017). Jarugula et al. (2018) proved for the first time that BSMV can be used as a vector for maize VIGS by knocking out endogenous host plant desaturase gene. Huang (2017) analyzed the functional genomics of wheat by using the BSMV-VIGS system and found some genes related to rust resistance. Gunupuru et al. (2019) proposed a barley BSMV-VIGS gene silencing scheme and described its role in resistance to Fusarium infection in barley leaves. Consequently, BSMV-VIGS has been widely used in monocotyledonous plants.

As an important component of the cytoskeleton, the microfilament cytoskeleton assembled by actin is involved in the occurrence of various plant cell processes, such as cytoplasmic flow, mitosis, and cell elongation (Hepler et al., 2001). The maintenance and change of microfilaments in cells depend on the action of actin binding proteins and regulatory proteins. Actin depolymerization factor (ADF) is a microfilament binding protein, which regulates the dynamics of microfilaments by promoting the treadmill movement of actin, thus affecting the occurrence and maintenance of cell morphology (Kanellos and Frame, 2016). ADFs are widely found in A. thaliana, tobacco, and wheat. AtADF4 regulates actin cytoskeleton dynamics in A. thaliana and plays an important role in controlling the resistance activation of genes and the signal transduction of mitogen-activated protein kinase (Porter et al., 2012). The overexpression of NtADF1 in tobacco can inhibit the mutation of NtRacl and lead to morphological changes of pollen tube (Chen et al., 2003). Common wheat TaADF7 is involved in the response to stem rust ( $\mathrm{Fu}$ et al., 2014). AtADF7 in A. thaliana participates in pollen development by cutting off actin filaments in pollen tubes to form longitudinal actin cords (Daher and Geitmann, 2012; Zheng et al., 2013). The effect of ADF7 silencing on the arrangement of microfilaments and plant growth has not been determined. Therefore, in the present study, wheat TaPDS and TaADF7 genes were silenced by BSMV-VIGS, and leaf bleaching was performed after TaPDS silencing to determine the feasibility of the silencing system. After further silencing of TaADF7, the arrangement of microfilaments in wheat mesophyll cells and the situation of plant growth were explored. This study will provide reference about the function of other genes in wheat.

\section{Materials and Methods}

Plant material and growth conditions

Yimai 325 (Triticum aestivum L.) seeds were supplied by Wheat Research Institute, Shanxi Academy of Agricultural Sciences, China. The seeds of Yimai 325 were selected and sterilized with $1 \% \mathrm{NaClO}$ for $10-15 \mathrm{~min}$ and then washed with sterilizing water for $10 \mathrm{~min}$. The seeds were placed in petri dishes covered with wet filter paper, each dish holding 30 seeds, and cultured in a growth chamber at $25^{\circ} \mathrm{C}$ and $60 \%$ relative humidity. After 7 days of growth, the fully expanded leaves were prepared for protoplast isolation.

The seeds of Nicotiana benthamiana were dropped into loose vegetative soil, incubated in a sealed container for approximately 2 weeks, and transplanted to produce tobacco seedlings with 3-4 leaves.
Strains and vectors

Escherichia coli DH5a, Agrobacterium GV3101, BSMV a, BSMV $\beta$, and BSMV $\gamma$ were obtained from Dawei Li Laboratory of China Agricultural University (Yuan et al., 2011). The cDNAs of BSMV $\alpha, \beta$, and $\gamma$ were inserted between the CaMV 35S double promoter and TRSV satellite RNA ribozyme sequence to obtain the corresponding vectors, and a LIC site was inserted, as shown in Fig. 1.

\section{Construction of vectors $p$ CaBS- $\gamma::$ TaPDS/TaADF7}

Total RNA from wheat leaves were extracted using Trizol reagent and then reverse transcribed into cDNA. The target genes TaPDS and TaADF7 were amplified with cDNA as template, detected by electrophoresis, and recovered by gel cutting. The $\gamma$ plasmids were extracted and ligated with TaPDS/TaADF7 in the presence of Apal mononuclease and T4 DNA polymerase. The recombinant plasmid was transformed into E. coli for colony PCR and sequencing identification. Agrobacterium tumefaciens was transformed after correct sequencing. The PCR primer sequences are shown in Tab. 1.

\section{RT-PCR of TaPDS and TaADF7}

(1) Trizol method was used to extract total RNA from wheat leaves;

(2) The extracted total RNA was reverse-transcribed into cDNA using PrimeScriptTM RT-PCR Kit;

(3) RT-PCR were performed on TaPDS and TaADF7 using CDNA as template. The reaction procedure of TaPDS: $95^{\circ} \mathrm{C} 2 \mathrm{~min}, 95^{\circ} \mathrm{C} 20 \mathrm{~s}, 58.4^{\circ} \mathrm{C} 20 \mathrm{~s}, 72^{\circ} \mathrm{C} 30 \mathrm{~s}, 72^{\circ} \mathrm{C} 5 \mathrm{~min}$, $4^{\circ} \mathrm{C}, 40$ cycles; the reaction procedure of TaADF7: $95^{\circ} \mathrm{C}$ $2 \mathrm{~min}, 95^{\circ} \mathrm{C} 20 \mathrm{~s}, 60.2^{\circ} \mathrm{C} 20 \mathrm{~s}, 72^{\circ} \mathrm{C} 30 \mathrm{~s}, 72^{\circ} \mathrm{C} 5 \mathrm{~min}, 4^{\circ} \mathrm{C}$, 40 cycles.

\section{Injected tobacco and rubbed wheat}

A. tumefaciens containing the plasmids were activated, shaken, and collected. The bacterial solution containing $p C a B S-\alpha$, $p C a B S-\beta$, and $p C a B S-\gamma:: T a P D S$ was mixed in a ratio of $1: 1: 1$, stood at $28^{\circ} \mathrm{C}$ for $3-5 \mathrm{~h}$ and then injected into tobacco with four leaves. The phenotypes of the injected and newly born leaves after 20 days of infection were observed. At 5-12 days after injection, tobacco leaves were ground in a buffer containing $1 \%$ diatomite and $0.1 \%$ sodium phosphate after sodium sulfite treatment. The juice was filtered through gauze and used to inoculate wheat (the wheat should be sprayed with emery before inoculation). $p C a B S-\gamma:: T a A D F 7$ was injected with tobacco and rubbed wheat in the same way. The height of wheat plant was measured using a ruler.

\section{Trypan blue staining}

Normal, dried, and $p C a B S-\gamma:: T a P D S$-treated wheat leaves were soaked in trypan blue dye solution for $1 \mathrm{~h}$ at approximately

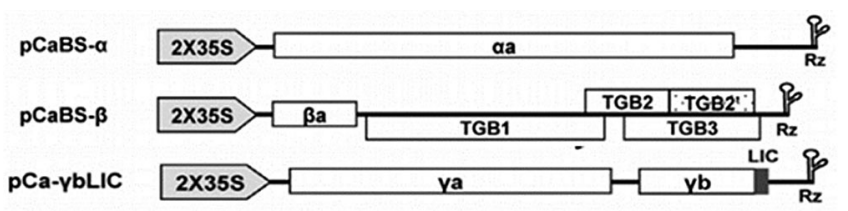

FIGURE 1. Schematic diagram of the construction of pCaBS- $\alpha$, $\mathrm{pCaBS}-\beta$, and $\mathrm{pCa}-\gamma \mathrm{bLIC}$ vector-induced gene silencing by BSMV. 
TABLE 1

Primer sequences of TaPDS and TaADF7

\begin{tabular}{lll}
\hline Primer & Sequence & Application \\
\hline TaPDS-F & AAGGAAGTTTAATTTCTCCAGGAGAAG & Target gene cloning \\
TaPDS-R & AACCACCACCACCGTCTGCATAAACGCTTAAAAG & Target gene cloning \\
TaADF7-F & AAGGAAGTTTAATGGCTGTGGACGACGAAT & Target gene cloning \\
TaADF7-R & AACCACCACCACCGTTGGCACGGACTTGGATGAC & Target gene cloning \\
TaPDS-F' & AAGGCTGGATAAGTTGGTGG & Colony PCR \\
TaPDS-R' & TGGTCTTCCCTTGGGGGAC & Colony PCR \\
TaADF7-F' & GGTGAGCCTGCCCTGAACT & Colony PCR \\
TaADF7-R' & TGGTCTTCCCTTGGGGGAC & Colony PCR \\
\hline Note: F forward primer R: reverse primer &
\end{tabular}

$1 \mathrm{~cm}$, and then decolorized overnight in $1.25 \mathrm{~g} / \mathrm{mL}$ chloral hydrate until the leaves became transparent. Then, microscope was used to record the leaves, and photos were captured.

\section{Preparation of protoplasts}

After seven days of growth, the wheat leaves were cut into $1 \mathrm{~mm}$ segments and treated with an enzyme solution containing $0.25 \%$ macerozyme R10 and $1 \%$ cellulase R10 in different W5 salt solutions $\left(154 \mathrm{mM} \mathrm{NaCl}, 125 \mathrm{mM} \mathrm{CaCl}_{2}, 5 \mathrm{mM} \mathrm{KCl}\right.$, and $2 \mathrm{mM}$ MES-KOH at $\mathrm{pH}$ 5.7) in different Petri dishes. The excised tissue was incubated in an incubator at $25^{\circ} \mathrm{C}$ for $3.5 \mathrm{~h}$. The incubated mixture was filtered through a $50 \mu \mathrm{m}$ nylon mesh and transferred to $10 \mathrm{~mL}$ centrifuge tubes. The sample was centrifuged at $500 \mathrm{rpm}$ for $5 \mathrm{~min}$, the supernatant was carefully removed, and $4 \mathrm{~mL}$ of $20 \%$ sucrose solution was added and mixed with protoplast suspension. The sample was gently covered with $2 \mathrm{~mL}$ of W5 saline solution without interfering with the protoplast suspension. The sample was centrifuged again at $500 \mathrm{rpm}$ for $5 \mathrm{~min}$. Floating protoplasts were collected using a sterile pipette, and a ring was formed between the W5 and sucrose solution layers. The protoplasts were diluted in W5 solution and centrifuged at $500 \mathrm{rpm}$ for $2 \mathrm{~min}$ (Chen and Han, 2016; Pitzschke and Persak, 2012; Rao and Prakash, 1995).

\section{Fluorescence staining of microfilaments}

FITC-Ph was bound specifically to F-actin in eukaryotic cells to reveal the distribution of microfilaments in cells. Wheat protoplasts were incubated at $37^{\circ} \mathrm{C}$ at $50 \mu \mathrm{g} / \mathrm{mL}$ FITC-Ph for $40 \mathrm{~min}$ and then observed (Zheng et al., 2013).

\section{Microscopic observation and data statistics}

Fluorescence observations were conducted using an FV-1000 confocal system with a $1.4 \mathrm{NA}, 60 \times$ oil immersion objective lens. The actin filaments were stained with FITC-Ph, and the excitation wavelength was $488 \mathrm{~nm}$. The captured images were $512 \times 512$ pixels in size. IBM SPSS software was used for data analysis.

\section{Results}

Identification of $p C a B S-\alpha / \beta / \gamma$ vectors and cloning of TaPDS/ TaADF7 genes

E. coli containing $p C a B S-\alpha, p C a B S-\beta$ and $p C a B S-\gamma$ vectors were activated and shaken. The plasmids $\alpha, \beta$, and $\gamma$ were extracted using the plasmid extraction kit, and then electrophoresis was performed with $1 \%$ agarose gel. The results are shown in Fig. 2. The sizes of $\alpha, \beta$ and $\gamma$ fragments were 3,787, 3,293, and $2,790 \mathrm{bp}$, respectively. The target genes TaPDS and TaADF7 were amplified and detected by electrophoresis. The results are shown in Fig. 2. The sizes of the amplified fragments of TaPDS and TaADF7 were 400 and $493 \mathrm{bp}$, respectively, which were correct. The $p C a B S-\gamma:: T a P D S /$ TaADF7 vectors were successfully obtained by enzymatic ligation with Apal mononuclease and T4 DNA polymerase.

\section{TaPDS silencing resulted in bleaching of wheat leaves}

Bacterial solutions containing $p C a B S-\alpha, p C a B S-\beta$, and $p C a B S$ $\gamma:: T a P D S$ were mixed in a ratio of $1: 1: 1$ and then injected into tobacco leaves by using the agrobacteria-mediated method. The phenotype of tobacco leaves after 20 days is shown in Fig. 3B. Light green areas appeared in the upper layer of infected tobacco leaves. The bacterial solution containing $B S M V:: 00$ was injected into tobacco leaves. The phenotype structure after 20 days is shown in Fig. 3A, and bleaching phenomenon did not occur in the leaves. The sap of infected leaves was rubbed against wheat at 5-12 days after tobacco injection, and the phenotype of wheat leaves at 25 days after inoculation is shown in Fig. 3C. In comparison with the WT and $p C a B S-\gamma:: 00$ treatment groups, wheat treated with $p C a B S-\gamma:: T a P D S$ showed significant bleaching phenomenon. Semi-quantitative PCR detection of TaPDS genes was conducted in wheat leaves under different treatment groups (Fig. 3D). Results showed no significant difference between the control and empty vector groups, and the bands in silenced group were weak. Therefore, the BSMV-VIGS system is feasible in wheat.

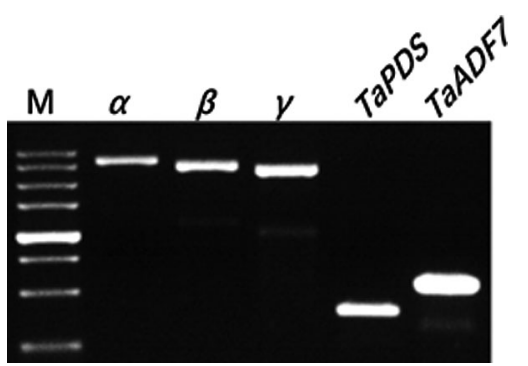

FIGURE 2. Electrophoretic patterns of $\alpha, \beta$, and $\gamma$ plasmids, TaPDS and TaADF7 genes. 


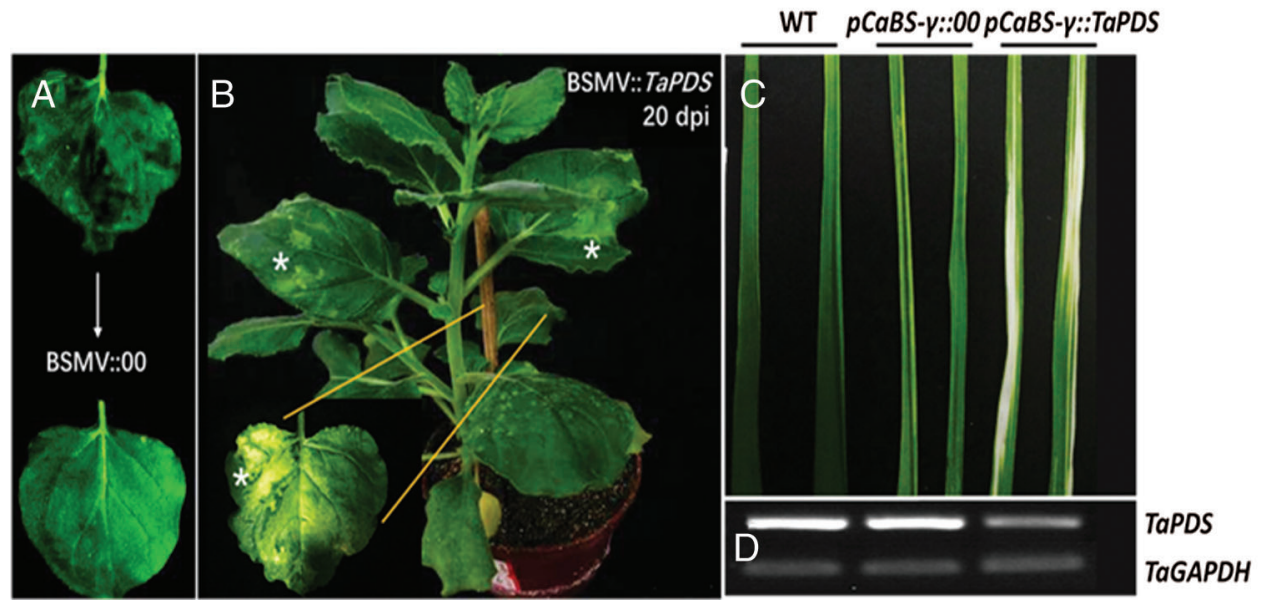

FIGURE 3. Phenotype of tobacco and wheat leaves after $p C a B S-\gamma:: T a P D S$ injection. (A) Phenotype of tobacco 20 days after inoculation with $B S M V:: 00$. Scale bar $=2 \mathrm{~cm}$. (B) Tobacco leaves at 20 days after $B S M V:: T a P D S$ injection. Scale bars = $2 \mathrm{~cm}$. (C) Phenotype of wheat leaves in WT, $p C a B S-\gamma:: 00$, and $p C a B S-\gamma::$ TaPDS groups after 25 days of inoculation. Scale bar $=5 \mathrm{~mm}$. (D) Semi-quantitative detection of TaPDS genes in wheat leaves under different treatment groups.

The cells in the bleached area of wheat leaves are living cells This study aimed to determine whether wheat leaves bleached after $p C a B S-\gamma:: T a P D S$ treatment withered. Trypan blue was used to stain the bleached leaves, and the results are shown in Fig. 4. The control (Figs. $4 \mathrm{~A}$ and $4 \mathrm{~B}$ ) group consisted of normal growing leaves, while $p C a B S-\gamma:: T a P D S$ (Figs. $4 \mathrm{E}$ and $4 \mathrm{~F}$ ) consisted of bleached leaves. Trypan blue staining results showed that the leaves could not be stained, indicating that the leaf cells in this group were living cells. However, the dried leaves treated at high temperature (Figs. 4C and 4D) were stained blue, indicating cell death in the dried leaves. For the wheat leaves were bleached by $p C a B S-\gamma:: T a P D S$, the cells remained alive, indicating that the BSMV-VIGS system was effective in the gene silencing of wheat.

Silencing TaADF7 resulted in the aggregation of microfilaments and inhibition of plant growth

The expression level of TaADF7 in wheat leaves after different treatments was detected using RT-PCR assay. The results are

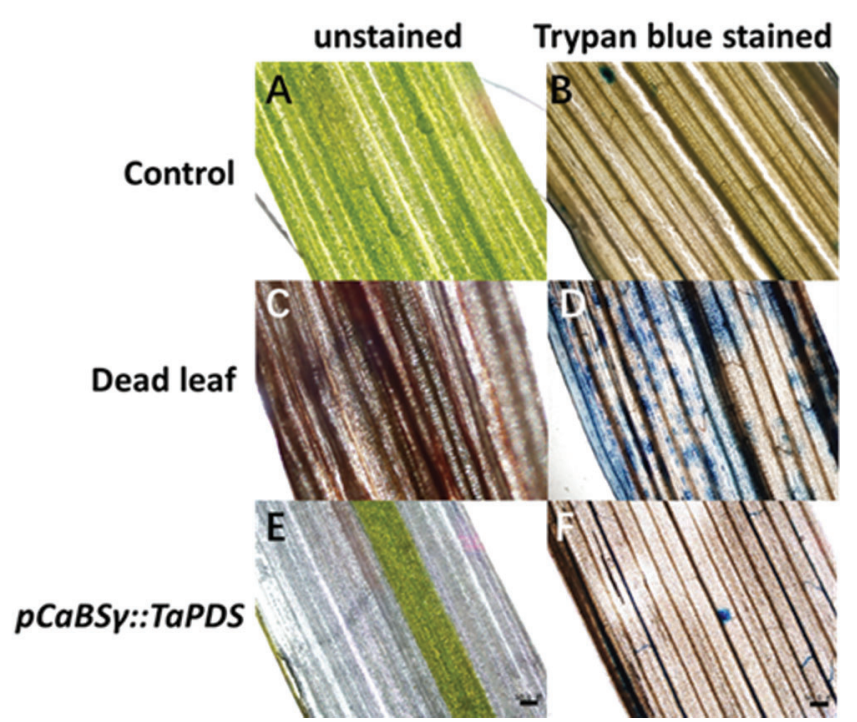

FIGURE 4. Identification of wheat leaf bleaching area by trypan blue staining. (A) Wheat leaf growing normally without staining. (B) Normal growing leaf stained with trypan blue. (C) Dead leaf without staining. (D) Dead leaf stained with trypan blue. (E) Unstained wheat leaf inoculated with $p C a B S-\gamma:: T a P D S$. (F) Wheat leaf inoculated with $p C a B S-\gamma:: T a P D S$ after trypan blue staining. Scale bars $=60 \mu \mathrm{m}$. shown in Figs. 5A and 5C. The expression level of TaADF7 in wheat leaves inoculated with $p C a B S-\gamma:: T a A D F 7$ was significantly reduced $(P<0.01)$ compared with WT group and the wheat leaves inoculated with $p C a B S-\gamma:: 00$, and this value decreased by $80 \%$ compared with WT control group, indicating that BSMV-VIGs successfully silenced TaADF7.

The protoplast microfilaments of leaves in different treatment groups were observed as shown in Figs. 5B and 5D. Fluorescence staining results showed that green fluorescence could be detected in wheat mesophyll protoplasts of different treatment groups, but the morphology of microfilaments varied greatly. Protoplast microfilaments in WT group and inoculated $p C a B S-\gamma:: 00$ group showed relatively diffuse state. However, the microfilaments in $p C a B S-\gamma:: T a A D F 7$ group showed an obvious filament-like structure. Fluorescence intensity analysis showed (Fig. 5D) no significant difference in terms of intracellular fluorescence intensity between different treatment groups. Hence, ADF7 silencing did not change the overall concentration of intracellular microfilaments, and the loss of ADF7 is reflected in changes in intracellular microfilament dynamics. The silencing of ADF7 caused intracellular microfilaments to aggregate into thick bundles rather than the diffuse structures as observed in the control group. Hence, TaADF7 is closely related to the dynamics of the microfilament skeleton, and ADF7 can regulate the dynamics of the microfilament skeleton.

The wheat plant height of WT, $p C a B S-\gamma:: 00$, and $p C a B S$ $\gamma:: T a A D F 7$ groups were measured, as shown in Fig. $5 \mathrm{E}$. The plant height of wheat inoculated with $p C a B S-\gamma:: 00$ was slightly lower than that of the control group $(P>0.05)$, while the plant height of wheat inoculated with $p C a B S-\gamma:$ : TaADF7 was significantly lower by approximately $80 \%$ than that of the control group $(P<0.01)$, indicating that the silencing of TaADF7 inhibited plant growth. This growth inhibition is associated with ADF7 silencing, which led to the aggregation of microfilaments in mesophyll cells into bundles and cannot be depolymerized in a timely manner, thus becoming diffused. Subsequently, the occurrence of cell dynamics was reduced as indicated by the growth inhibition.

\section{Discussion}

Chemical or radiation mutagenesis, insertion mutation, and RNAi can all achieve gene knockout or silencing in 


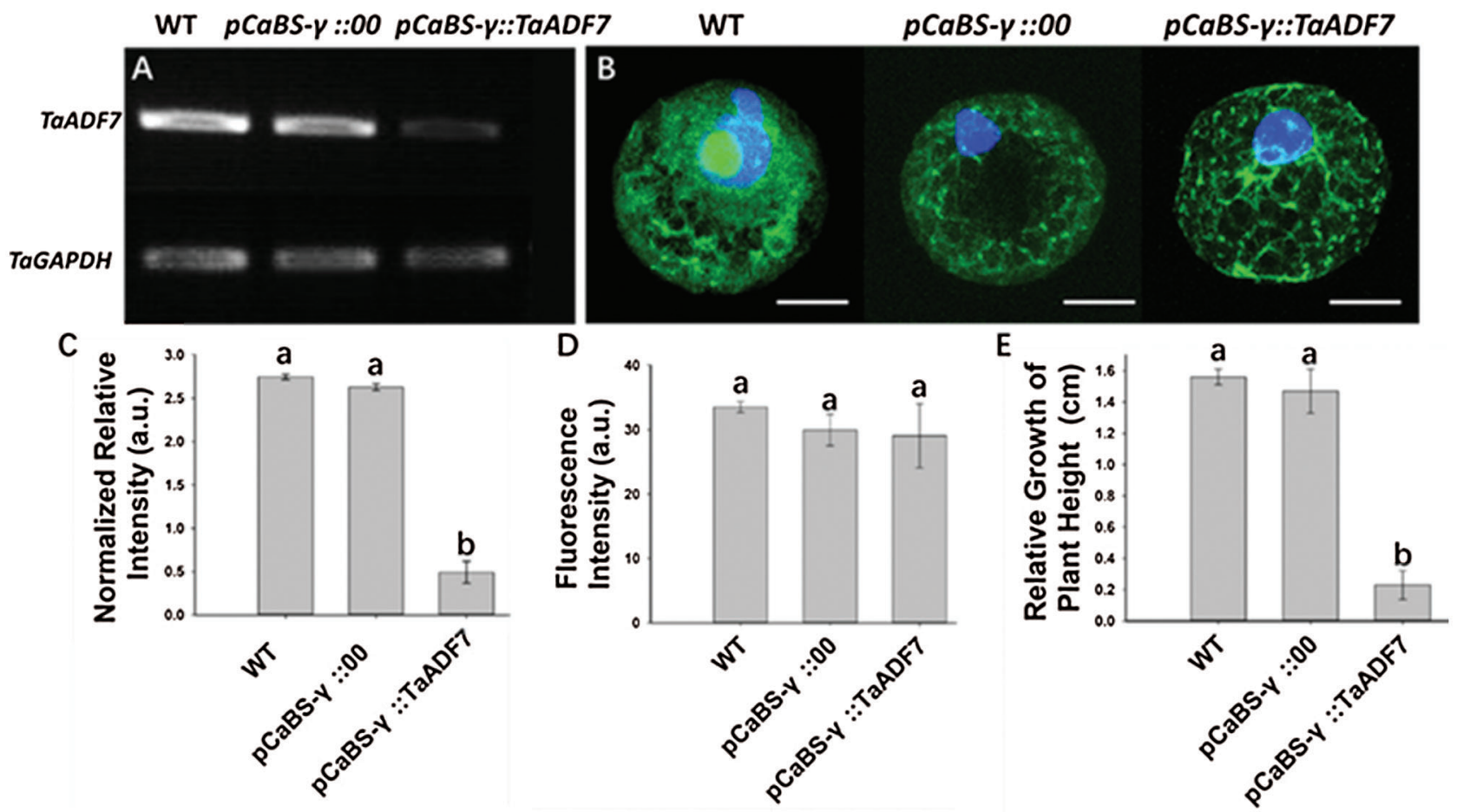

FIGURE 5. Expression level of ADF7 in wheat leaves, fluorescence intensity of actin cytoskeleton and plant height in different treatment groups. (A) RT-PCR analysis of TaADF7 in WT group, wheat leaf group inoculated with $p C a B S-\gamma:: 00$, and wheat leaf group inoculated with $p C a B S-\gamma:: T a A D F 7$. (B) Fluorescence observation of microfilaments in the wheat leaf protoplasts of different treatment groups. Scale bars $=10 \mu \mathrm{m}$. (C) TaADF7 expression in the wheat leaves of three treatment groups. (D) Fluorescence intensity analysis of microfilaments in the wheat leaf protoplasts of different treatment groups. (E) Measurement results of wheat plant height in different treatment groups.

monocotyledons and dicotyledons, but these methods require a long transformation process, while VIGS can be independent of stable genetic transformation and rapid identification of gene function. This method may also silence single or multiple genes in a gene family (SenthilKumar et al., 2008; Becker and Lange, 2010; Cakir et al., 2010; Tavakol, 2018), especially in wheat, an important crop of Gramineae with complex genomes.

PDS is a key gene in carotenoid synthesis, and its silence will block the carotenoid synthesis and decomposition of chlorophyll without protection, block photosynthesis, and cause subsequent bleaching in plants (Qin et al., 2007). The leaves of tobacco infected with $p C a B S-\gamma::$ TaPDS were ground into juice and then rubbed into wheat, and the wheat leaves were bleached. Trypan blue staining indicated that the bleached cells were biologically active cells instead of dead cells. Hence, the VIGS system has a great application prospect in the study of wheat gene function because the occurrence of bleaching indicates that the VIGS system is highly efficient in silencing wheat genes. PDS has been used as a marker gene to detect the occurrence of leaf bleaching in Brachypodium brachypodium and barley when the VIGS system was applied (Pacak et al., 2010; Yuan et al., 2011; Scofield et al., 2005; Hein et al., 2005). Hence, VIGS also has a high efficiency in the study of gene function in monocotyledonous gramineae.

ADF is a highly conserved actin depolymerizing factor in eukaryotic cells (Poukkula et al., 2011; Xu et al., 2021), and it preferably binds to ADP-actin, resulting in the loss of subunits at the tip of the microfilaments and improving the efficiency of the microfilament occurrence of the pedal phenomenon
(Maciver and Hussey, 2002; Bamburg et al., 1999). In vitro experiments have shown that TaADF can bind to actin and cut microfilaments (Fu et al., 2014). Microfilaments are associated with chromosomal localization in wheat cells during mitosis and possibly they may affect hypocotyl elongation by dynamically regulating themselves in response to UV-B radiation and $\mathrm{H}_{2} \mathrm{O}_{2}$ signals. Moreover, microfilaments may affect processes, such as plant growth and cell elongation. As an important factor in the regulation of microfilament dynamics, ADF7 may affect plant height by affecting microfilament dynamics. As shown in Fig. 5E, the relative elongation of plant height in the ADF7 gene silencing group was significantly lower than that in the control and empty vector group. Based on Fig. 5D, the overall fluorescence intensity in the cells was not significantly different, but as shown in Fig. 5B, the morphology of the microfilaments changed. Hence, the dynamic degree of intracellular microfilaments was reduced after ADF7 deletion, and the morphology of microfilament bundles was thick, and these conditions not good for cell elongation or growth. Therefore, at the plant level, the relative elongation of the plant is inhibited.

\section{Conclusion}

In the present study, the BSMV-VIGS system was used to silence wheat TaPDS and TaADF7 genes. Bleaching phenomenon was observed in wheat leaves after TaPDS silencing. After TaADF7 silencing, microfilaments in wheat mesophyll cells gathered into coarse bundles, which affected the dynamics of microfilaments and inhibited plant growth. 
Acknowledgement: Funders are thanked for support of this writing.

Availability of Data and Materials: All data generated or analysed during this study are included in this article.

Author Contribution: Chen $\mathrm{H}$, Ran $\mathrm{H}$ conceived and designed the experiments; Jia $\mathrm{X}$ and Niu J performed most of the experiments; Ran $\mathrm{H}$ and $\mathrm{Du} \mathrm{M}$ assisted; Chen $\mathrm{H}$, Jia $\mathrm{X}$, and $\mathrm{Du} \mathrm{M}$ wrote the paper and revised the article.

Ethics Approval: No committees were required for this study.

Funding Statement: This study was supported by Natural Science Foundation of China (NSFC31900251) and Postgraduate Course in Dual Language Foundation of Shanxi Normal University (YJSSY201902).

Conflicts of Interest: The authors declare that they have no conflicts of interest to report regarding the present study.

\section{References}

Bamburg JR, McGough A, Ono S (1999). Putting a new twist on actin: ADF/cofilins modulate actin dynamics. Trends in Cell Biology 9: 364-370.

Barciszewska-Pacak M, Jarmołowski A, Pacak A (2016). Virusinduced gene silencing for gene function studies in barley. Methods in Molecular Biology 1398: 293-308.

Becker A, Lange M (2010). VIGS-genomics goes functional. Trends in Plant Science 15: 1-4.

Cakir C, Gillespie ME, Scofield SR (2010). Rapid determination of gene function by virus-induced gene silencing in wheat and barley. Crop Science 50: S77-S84.

Chen CY, Cheung AY, Wu HM (2003). Actin-depolymerizing factor mediates Rac/Rop GTPase-regulated pollen tube growth. Plant Cell 15: 237-249.

Chen H, Han R (2016). Characterization of actin filament dynamics during mitosis in wheat protoplasts under UV-B radiation. Scientific Reports 6: 20115.

Daher FB, Geitmann A (2012). Actin depolymerizing factors ADF7 and ADF10 play distinct roles during pollen development and pollen tube growth. Plant Signaling \& Behavior 7: 879-881.

El Baidouri M, Murat F, Veyssiere M, Molinier M, Flores R et al. (2017). Reconciling the evolutionary origin of bread wheat (Triticum aestivum). New Phytologist 213: 1477-1486.

Fu Y, Duan X, Tang C, Li X, Voegele RT et al. (2014). TaADF7, an actin-depolymerizing factor, contributes to wheat resistance against Puccinia striiformisf. sp. tritici. Plant Journal 78: $16-30$.

García-Molina MD, Giménez MJ, Sánchez-León S, Barro F (2019). Gluten free wheat: Are we there? Nutrients 11: 487.

Gunupuru LR, Perochon A, Ali SS, Scofield SR, Doohan FM (2019). Virus-Induced Gene Silencing (VIGS) for functional characterization of disease resistance genes in barley seedlings. Methods in Molecular Biology 1900: 95-114.

Hein I, Barciszewskapacak M, Hrubikova K, Williamson S, Dinesen M et al. (2005). Virus-induced gene silencing-based functional characterization of genes associated with powdery mildew resistance in barley. Plant Physiology 138: 2155-2164.

Hepler PK, Vidali L, Cheung AY (2001). Polarized cell growth in higher plants. Annual Review of Cell and Developmental Biology 17: 159-187.
Huang L (2017). BSMV-induced gene silencing assay for functional analysis of wheat rust resistance. Methods in Molecular Biology 1659: 257-264.

Jarugula S, Willie K, Stewart LR (2018). Barley stripe mosaic virus (BSMV) as a virus-induced gene silencing vector in maize seedlings. Virus Genes 54: 616-620.

Kanellos G, Frame MC (2016). Cellular functions of the ADF/cofilin family at a glance. Journal of Cell Science 129: 3211-3218.

Lee WS, Rudd JJ, Kanyuka K (2015). Virus induced gene silencing (VIGS) for functional analysis of wheat genes involved in Zymoseptoria tritici susceptibility and resistance. Fungal Genetics and Biology 79: 84-88.

Maciver SK, Hussey PJ (2002). The ADF/cofilin family: Actinremodeling proteins. Genome Biology 3: 1-12.

Pacak A, Geisler K, Jørgensen B, Barciszewska-Pacak M, Nilsson L et al. (2010). Investigations of barley stripe mosaic virus as a gene silencing vector in barley roots and in Brachypodium distachyon and oat. Plant Methods 6: 26.

Pitzschke A, Persak H (2012). Poinsettia protoplasts-a simple, robust and efficient system for transient gene expression studies. Plant Methods 8: 14.

Porter K, Shimono M, Tian M, Day B (2012). Arabidopsis actindepolymerizing factor-4 links pathogen perception, defense activation and transcription to cytoskeletal dynamics. PLoS Pathogens 8: e1003006.

Poukkula M, Kremneva E, Serlachius M, Lappalainen P (2011). Actin-depolymerizing factor homology domain: A conserved fold performing diverse roles in cytoskeletal dynamics. Cell Motility and the Cytoskeleton 68: 471-490.

Qin G, Gu H, Ma L, Peng Y, Deng XW, Chen Z, Qu LJ (2007). Disruption of phytoene desaturase gene results in albino and dwarf phenotypes in Arabidopsis by impairing chlorophyll, carotenoid, and gibberellin biosynthesis. Cell Research 17: 471-482.

Ramegowda V, Mysore KS, Senthil-Kumar M (2014). Virus-induced gene silencing is a versatile tool for unraveling the functional relevance of multiple abiotic-stress-responsive genes in crop plants. Frontiers in Plant Science 5: 323.

Rao KS, Prakash AH (1995). A simple method for the isolation of plant protoplasts. Journal of Biosciences 20: 645-655.

Scofield SR, Huang L, Brandt AS, Gill BS (2005). Development of a virus-induced gene-silencing system for hexaploid wheat and its use in functional analysis of the lr21-mediated leaf rust resistance pathway. Plant Physiology 138: 2165-2173.

Senthil-Kumar M, Mysore KS (2011). New dimensions for VIGS in plant functional genomics. Trends in Plant Science 16: 656-665.

Senthil-Kumar M, Rame Gowda HV, Hema R, Mysore KS, Udayakumar M (2008). Virus-induced gene silencing and its application in characterizing genes involved in waterdeficit-stress tolerance. Plant Physiology 165: 1404-1421.

Tavakol E (2018). Virus-Induced Gene Silencing (VIGS) in Aegilops tauschii and its use in functional analysis of AetDREB2. Molecular Biotechnology 60: 41-48.

Unver T, Budak H (2009). Virus-induced gene silencing, a post transcriptional gene silencing method. International Journal of Plant Genomics 2009: 198680.

Wang M, Wang S, Xia G (2015). From genome to gene: A new epoch for wheat research? Trends in Plant Science 20: 380-387.

Xu K, Zhao Y, Zhao S, Liu H, Wang W, Zhang S, Yang X (2021). Genome-wide identification and low temperature responsive pattern of Actin Depolymerizing Factor (ADF) 
gene family in wheat (Triticum aestivum L.). Frontiers in Plant Science 12: 618984.

Yuan C, Li C, Yan L, Jackson AO, Liu Z et al. (2011). A high throughput barley stripe mosaic virus vector for virus induced gene silencing in monocots and dicots. PLoS One 6: e26468.

Zeng H, Xie Y, Liu G, Wei Y, Hu W, Shi H (2019). Agrobacteriummediated gene transient overexpression and Tobacco Rattle Virus (TRV)-based gene silencing in cassava. International Journal of Molecular Sciences 20: 3976.
Zhang J, Yu D, Zhang Y, Liu K, Xu K et al. (2017). Vacuum and cocultivation agroinfiltration of (germinated) seeds results in Tobacco Rattle Virus (TRV) mediated whole-plant VirusInduced Gene Silencing (VIGS) in wheat and maize. Frontiers in Plant Science 8: 393.

Zheng Y, Xie Y, Jiang Y, Qu X, Huang S (2013). Arabidopsis actindepolymerizing factor7 severs actin filaments and regulates actin cable turnover to promote normal pollen tube growth. Plant Cell 25: 3405-3423. 